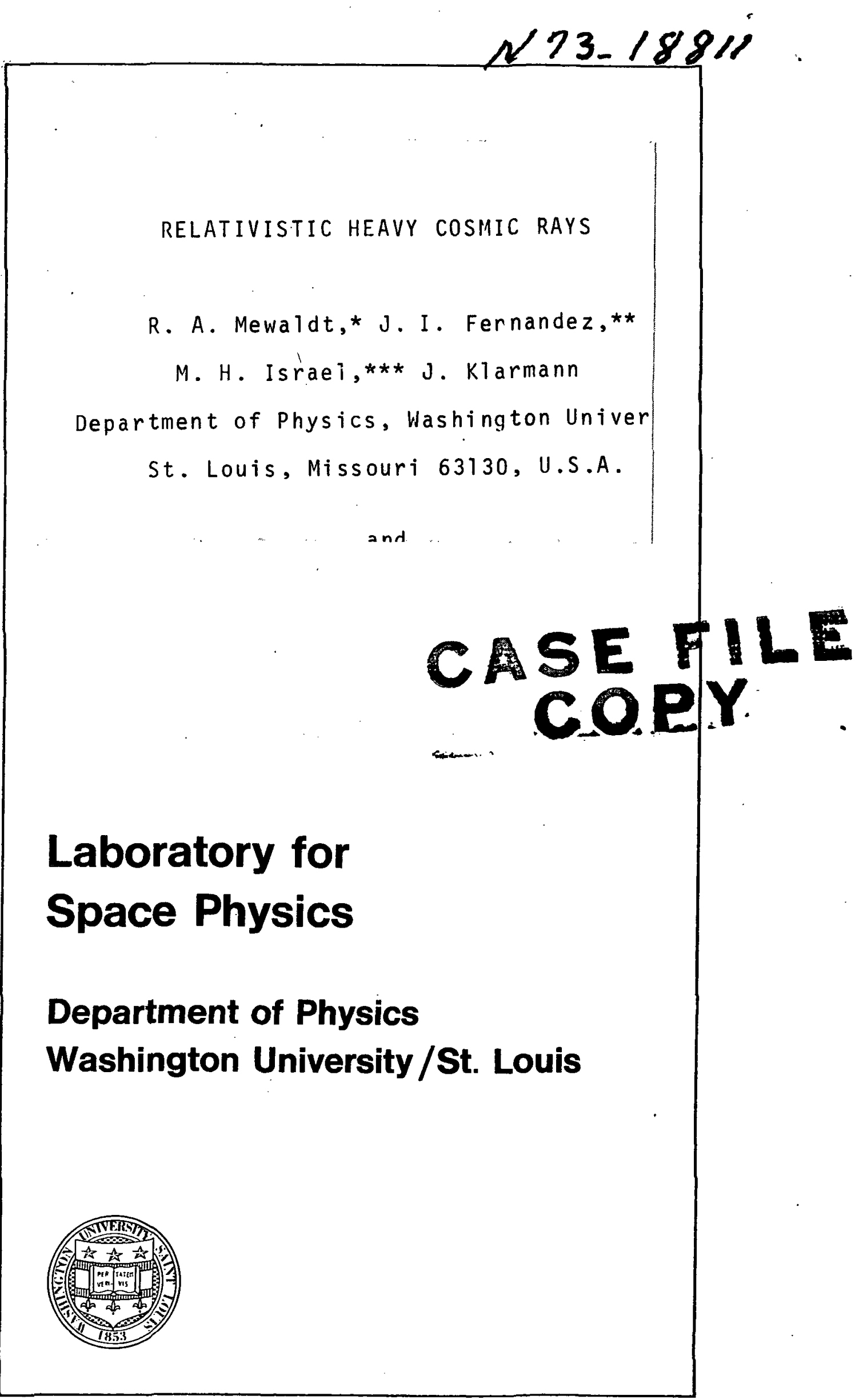




\title{
RELATIVISTIC HEAVY COSMIC RAYS
}

\author{
R. A. Mewaldt,* J.I. Fernandez,** \\ M. H. Israe $1, * * *$ J. Klarmann \\ Department of Physics, Hashington University \\ St. Louis, Missouri 63130, U.S.A. \\ and \\ W. R. Binns \\ McDonnel1 Douglas Research Laboratories \\ St. Louis, Missouri 63166, U.S.A.
}

*Present Address: Downs Laboratory of Physjcs,

California Institute of Technology,

Pasadena, California 91109 , U.S.A.

**Present Address: Department of Physics, Makerere University, Kampala, Uganda

$\star \star \star A l f r e d P$. Sloan Research Fellow 


\section{ABSTRACT}

During three balloon flights of a $1 \mathrm{~m}^{2}$ sr ionizationchamber/Cerenkov counter detector: system, we have measured the atmospheric attenuation, $f 1 u x$, and charge composition of cosmic-ray nuclei with $16 \leq Z \leq 30$ and rigidity greater than $4.5 \mathrm{GV}$.

The attenuation mean-free-path in air of $V H(20 \leq Z \leq 30)$ nuclei is found to be $19.7 \pm 1.6 \mathrm{~g} / \mathrm{cm}^{2}$, a value somewhat greater than the best previous measurement. The attenuation mean-free-path of iron is found to be $15.6 \pm 2.2 \mathrm{~g} / \mathrm{cm}^{2}$, consistent with predictions of geometric cross-section formulae.

We measure an absolute flux of VH nuclei 10 to $20 \%$ higher than earlier experiments at similar geomagnetic cutoff and level of solar activity. The relative abundances of evencharged nuclei are found to be in good agreement with results of other recent high-resolution counter experiments.

We calculate that our observed cosmic ray chemical composition implies relative abundances at the cosmic-ray source of $\mathrm{Ca} / \mathrm{Fe}=0.12 \pm 0.04$ and $\mathrm{S} / \mathrm{Fe}=0.14 \pm 0.05$. The results are consistent with all other elements of charge between 16 and 26 being absent at the source and being produced by cosmic-ray fragmentation in intersteilar hydrogen. The results show a cosmic-ray source deficient relative to the solar system in both sulfur and argon. 


\section{INTRODUCTION}

We have measured the flux and composition of cosmic-ray nuclei with atomic number ( $Z$ ) greater than 15 and rigidity greater than $4.5 \mathrm{GV}$. These measurements were made in a series of three balloon flights during 1970 with a large-area detector using ionization chambers and a cerenkov counter. In this paper we present results on nuclei with $16 \leq Z \leq 30$. Our measurements of VVH $(Z>30)$ nuclei will be presented in a later publication.

With our very large geometrical factor (0.92 $\left.\mathrm{m}^{2} \mathrm{ster}\right)$, we have observed more than 25,000 VH $(20 \leq Z \leq 30)$ nuclei, several times the sum of all previous investigations. Although our charge resolution is not as good as that of some other investigators (Webber et al., 1971; Casse et al., 1971) we have used our excellent statistics to measure properties not easily accessible to these other experiments, including the atmospheric attenuation of these nuclei, and their absolute flux. In addition we have measured relative abundances of even- $Z$ nuclei and extrapolated these abundances back to the cosmic ray source. Our calculations demonstrate that either a simple slab model or a steady-state exponentialpath-length model of cosmic-ray propagation can fit the observed abundances of nuclei with $16 \leq z \leq 30$ with nearly the same source abundances. 
Preliminary results of these three flights and of a later flight at lower geomagnetic cutoff have been presented at the Twelfth International Conference on Cosmic Rays (Binns et al., 1971). Further detail on this experiment and the data analys is may be found in Mewaldt, (1971), and a more detailed detector system description appears in Epstein et al., (1971). 


\section{THE DETECTOR SYSTEM AND BALLOON FLIGHTS}

A schematic diagram of the detector system is shown in Figure 1. The principal components are the two pulse-type ionization counters and the Lucite Cerenkov counter. The two parallel-plate ionization counters are $1 \mathrm{~m}^{2}$ in area, $5.1 \mathrm{~cm}$ thick, and filled with a mixture of $90 \%$ argon and $10 \%$ methane at a pressure of one atmosphere. The Cerenkov radiator is a $1 \mathrm{~m}^{2}$ sheet of $0.6-\mathrm{cm}$ thick ultraviolet-transmitting (UVT), Lucite, with light collection by total internal reflection. Each of the four edges of the radiator is viewed by a $12.7-\mathrm{cm}$ photomultiplier (RCA 4525) through an adiabatic light pipe constructed of UVT Lucite. The outputs of the four phototubes are summed. Approximately 2.5 photoelectrons result from the perpendicular traversal of a relativistic single-charged particle.

The particle trajectories are given by a hodoscope consisting of two crossed layers of scintillator strips above the other counters, and two crossed layers below. Each layer consists of ten strips, giving $9.5 \mathrm{~cm}$ resolution. A six-fold coincidence among the four hodoscope layers and the two ion chambers triggers the recording of an event. The ion chamber discriminators determine the experiment threshold and are typically set at a signal level corresponding to a vertically incident relativistic nucleus of charge 16. For each event, 
the signals from each of the ion chambers and from the cerenkov counter are pulse height analysed, and the trajectory of the particle is indicated by recording which hodoscope-strip discriminators were triggered.

There are four guard counters, one on each side of the detector square. The guard counters are not in active anticoincidence, but the outputs of the four guard counter discriminators are recorded for each particle triggering the system.

This paper treats data gathered in three high altitude balloon flights of this detector system (Table I). The residual atmosphere, geomagnetic cutoff, and neutron monitor values averaged over the entire float are presented here for comparison; however, in applying corrections to the data, hourly averages were used. 


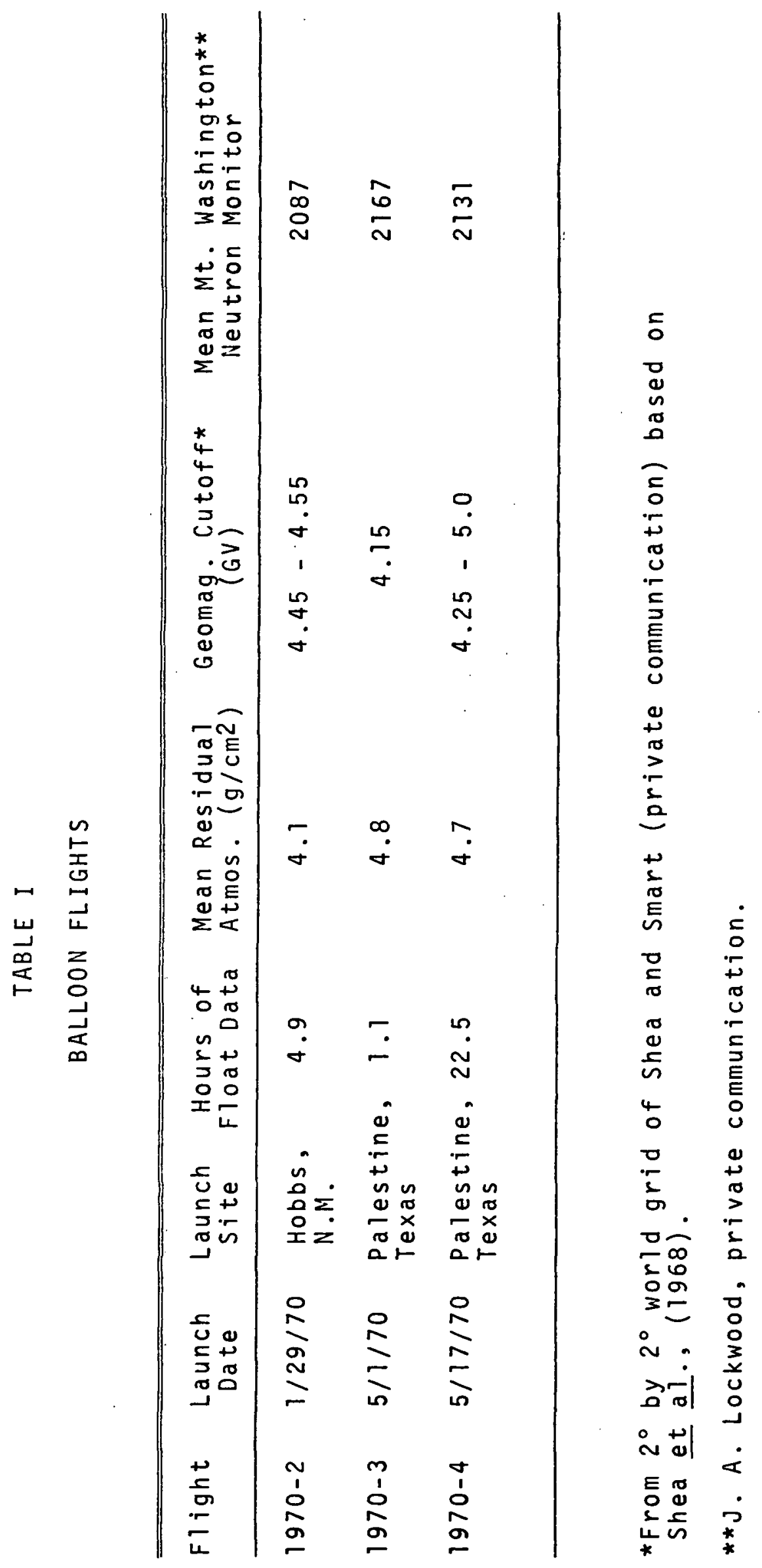




\section{CORRECTIONS TO RAW DATA}

The raw data were modified by correcting recorded pulse heights for temporal and spatial variations in detector response and by eliminating the small fraction of ambiguous events .

Three corrections were applied to the ionization chamber pulse heights. Corrections for variations of the temperature and pressure of the gas were made using pulse height spectra from the internal Americium-241 alpha-particle calibration source. Hodoscope data indicating position in the chambers of each particle's trajectory were used to correct for areal non-uniformities in the chamber response (typically 1 to $2 \%$ ). Hodoscope data indicating zenith angle $(\theta)$ of each particle's trajectory permitted correction of each pulse height for the sec $\theta$ dependence of the path length in the gas.

The Cerenkov counter pulse heights were corrected for non-uniformities in light collection which were determined empirically using cosmic-ray iron nuclei. The zenith angle dependence of Cerenkov pulse height was much stronger than $\sec \theta$, in general agreement with the performance of a similar counter described by Ormes and Balasubrahmanyan (1970). More significantly, the variation of light collection with velocity is considerably greater than the variation of light production. In addition, there are systematic azimuthal variations. We can explain the measured response of the counter in terms of 
the amount of light produced, the fraction that is internally reflected, and a semiempirically-determined light collection efficiency (Mewaldt, 1971).

In selecting events for further analysis, we have eliminated only those events for which charge identification was highly ambiguous. Our criteria rejected $4 \%$ of all recorded events. Appropriate small corrections were made in determining absolute fluxes. In eliminating the ambiguous events we avoided criteria which might preferentially eliminate some charges. The acceptance criteria were:

1. Ion Chamber Agreement--Events in which the two ion chambers disagreed by more than three charge units were rejected. The number of such events $(\sim 1 \%)$ is consistent with the expected number of nuclear interactions in the material between the chambers.

2. Ion Chamber-Cerenkov Consistency--Events were rejected if the ratio of Cerenkov to ionization signals was more than three standard deviations greater than the maximum theoretical value. These were less than $0.2 \%$ of all events.

3. Multiple Hodoscope Events--In about $7.5 \%$ of all events, two adjacent hodoscope strips were triggered in at least one hodoscope layer, in many cases the result of knockon electrons. These events were normally accepted. However, when two non-adjacent strips were triggered, $(\sim 2.5 \%$ of a 11 events) the trajectory was ambiguous, and the events were rejected. 
4. Guard Events--One or more guard counters was triggered in less than $2 \%$ of the events. Since these events appear to be normal in other respects, they were rejected only if two or more guards fired, or a simultaneous multiple hodoscope occurred, amounting to less than $0.5 \%$ of all events.

We note that the $4 \%$ event rejection is significantly less than the fraction of events that undergo nuclear interactions within the detector system. Indeed, many of these interactions are not distinguishable from normal events. At the energies of the particles detected in these flights, interaction secondaries closely preserve the direction of the primary nucleus, and our relatively coarse hodoscope resolution does not resolve the individual fragments. To correct for interactions in the material above the ion chambers $\left(1.2 \mathrm{~g} / \mathrm{cm}^{2}\right)$, we extrapolated the observed spectrum through an equivalent thickness of atmosphere. Interactions below the ion chambers have almost no effect on the charge assignment, as it is determined almost solely by the ion chamber measurements. 


\section{PARTICLE IDENTIFICATION}

Both the rate of energy-loss of a particle (measured by the ionization chamber) and its Cerenkov light production are proportional to $Z^{2}$, but the two quantities have different functional dependence on the particle's velocity, so it is possible to use these measurements to determine both the particle's charge and velocity. Figure 2 shows a cross-plot of the square root of the ionization $(\sqrt{\mathrm{I}})$ vs the square root of the Cerenkov light intensity $(\sqrt{c})$ for even-z nuclei. We have included the measured response of our ionization counters in the relativistic rise region (Fernandez, 1972) and the response of our Cerenkov counter at $\theta=30^{\circ}$. For the balloon flights reported here, the events are expected to fall to the right of the 4.5 GV geomagnetic cutoff indicated on the crossplot. Note that charge and energy identification are ambiguous in the relativistic rise region where, for example, an iron nucleus of $20 \mathrm{GeV} / \mathrm{nucle}$ on produces the same I and $\mathrm{C}$ as a $2.0 \mathrm{GeV} /$ nucleon nickel nucleus. Because of the steepness of the cosmic ray energy spectrum, this ambiguity is a problem. only when the lower charge is much more abundant than the higher charge.

While it is apparent that for high cutoff flights, the Cerenkov counter provides only limited improvement over charge identification based on ionization alone, the Cerenkov counter 
is very useful in insuring that rare particles of very high ionization are actualiy relativistic VVH nuclei, and not slow VH nuclei.

Figure 3 shows a $\sqrt{I}$ vs $\sqrt{C}$ cross-plot for about 2000 events with $20 \leq \theta \leq 50^{\circ}$. The predicted response of the counters agrees well with the data. Notice that there are virtually no events lying significantly to the right (high C/I ratio) of the predicted response. There are, however, a substantial number of events $(\sim 6 \%)$ that appear to have energies less than that allowed by the geomagnetic cutoff (low $\mathrm{C} / I$ ratio). These events originate from particles that undergo nuclear interactions in the $0.7 \mathrm{~g} / \mathrm{cm}^{2}$ of the Lucite Cerenkov counter after passing through the ion chambers.

Because ionization is nearly independent of energy for rigidity greater than $4.5 \mathrm{GV}$, a good first approximation to charge identification is given by $Z \propto \sqrt{I}$. An improvement in charge resolution of about $20 \%$ was achieved for events due to particles with energy between 1 and $2 \mathrm{GeV} /$ nucleon where the ratio $\mathrm{C} / \mathrm{I}$ gives an unambigous, charge-independent measure of energy. However, for events with low Cerenkov signals, and those in the relativistic rise region ( $T>2 \mathrm{GeV}$ / nucleon), a velocity determination becomes impossible, and charge identification was based on ionization alone, using the mean of the two ion chambers. 
Figure 4 shows a charge spectrum obtained from $\sim 10,000$ events with $\theta<30^{\circ}$, collected at an atmospheric depth of $4.5 \mathrm{~g} / \mathrm{cm}^{2}$ and a vertical geomagnetic cutoff near $4.5 \mathrm{GV}$. The iron peak resolution ( 1.5 charge units $f(w h)$ is typical of the charge resolution obtained in this experiment.

The charge scale in Fig. 4, derived by assuming that the prominent peak is centered at iron, agrees to within 0.1 charge units with a calculated scale based on the ionization chamber response to the in-flight alpha-particle calibration source (Epstein et al., 1971). This agreement confirms the generally accepted assumption that the cosmic ray flux has a significant peak at $Z=26$.

In order to identify the relative contributions of the individual elements to this charge spectrum, a least squares fit was performed. The fit used a calculated pulse-height distribution for any individual element which took into account the measured response of our ion chambers in the relativistic-rise region and left the charge resolution as a free parameter. The resulting fit gave unambiguous results for the relative abundances of elements of even charge. The elements of odd charge are less abundant, and our resolution did not permit determination of their abundances. Nickel and $Z$ inc $(Z=28$ and 30$)$, though of even charge, were obscured by the relativistic rise of the much more abundant iron, so their abundances were not well determined. 


\section{ATtENUATION AND FRAgMENTATION IN THE ATMOSPHERE}

Taking advantage of our large geometrical factor, we have measured the atmospheric attenuation of VH nuclei using data from the ascent portions of the balloon flights. Charge spectra from each flight were obtained in each of seven depth intervals over the range $4.0 \leq x \leq 22.5 \mathrm{~g} / \mathrm{cm}^{2}$. The thickness of atmosphere penetrated by each incident particle was determined from its trajectory in the detector and the altitude of the balloon at the time of arrival; thus particles penetrating any given thickness of atmosphere were observed from various zenith angles at various balloon altitudes. The effective area $x$ solid-angle $x$ time factor for each depth interval was calculated by numerical integration of the detector's differential geometry factor over the balloon ascent trajectory. The data from the three flights were normalized to a $4.5 \mathrm{GV}$ vertical geomagnetic cutoff, assuming a $\mathrm{P}^{-1.4}$ integral rigidity spectrum, and to a Mt. Washington neutron monitor rate of 2128 using the helium regression curve of Webber (1967).

Figure 5 shows the depth dependences of the $F e$ and of the $V H$ fluxes along with the results of linear least squares fits. The data points have been plotted at the mean atmospheric depth traversed within each depth interval, as computed from the experimental data. Effects due to variation of geomagnetic cutoff with incident direction are negligible 
because each data point represents an average over all azimuths and a wide range of zenith angles.

The fit to the data yields an attenuation mean free path for iron of $\Lambda_{\mathrm{Fe}}=15.6 \pm 2.2 \mathrm{~g} / \mathrm{cm}^{2}$, in good agreement with the interaction mean free paths computed from the semi-empirical formulae of Daniel and Durgaprasad (1962), $14.2 \mathrm{~g} / \mathrm{cm}^{2}$; Bradt and Peters (1950), $14.0 \mathrm{~g} / \mathrm{cm}^{2}$; and Cleghorn et al. (1968), $15.5 \mathrm{~g} / \mathrm{cm}^{2}$. Our value for $\Lambda_{V H}\left(19.7 \pm 1.8 \mathrm{~g} / \mathrm{cm}^{2}\right)$ is noticeably greater than the value of $16.5 \pm 2 \mathrm{~g} / \mathrm{cm}^{2}$ obtained by Webber and Ormes (1967), although the uncertainties overlap.

The value of $\Lambda_{V H}$ is greater than $\Lambda_{\mathrm{Fe}}$ primarily because of the non-zero probability of. VH nucleus remaining in the $\mathrm{VH}$ group after an interaction $\left(\mathrm{p}_{V H}, V_{H}>0\right)$. Neglecting small differences in the geometrical cross sections of iron nuclei and $V H$ nuclei, we calculate

$$
p_{V H, V H} \quad 1-\frac{\Lambda_{F e}}{\Lambda_{V H}}=0.21 \pm 0.13
$$

This is consistent with the value $0.26 \pm 0.05$ obtained by Waddington (1960).

Extrapolation to the top of the atmosphere of fluxes of nuclei with $Z<26$ cannot assume a simple exponential attenuation, because these lighter nuclei are produced copiously in the atmosphere from iron fragmentation. It is convenient, instead, to extrapolate the ratio $R(x)=$ $N_{2}(x) / N_{1}(x)$ where $N_{i}$ is the flux of nuclei in group $i$ and 
$x$ is the atmospheric depth. If group 1 (e.g. iron) has negligible contribution from atmospheric fragmentation of heavier nuclei and group 2 consists of lighter nuclei whose only significant fragmentation source is group 1 , it can be shown (Mewaldt, 1971) that $R(x)$ varies linearly with $x$ for $x \ll \Lambda_{1} \Lambda_{2} /\left(\Lambda_{2}-\Lambda_{1}\right)$ as

$$
R(x)=R(0)+\left[R(0)+\frac{p_{12} \Lambda_{2}}{\Lambda_{2}-\Lambda_{1}}\right]\left[\frac{\Lambda_{2}-\Lambda_{1}}{\Lambda_{2} \Lambda_{1}}\right] x
$$

where $\Lambda_{i}$ is the attenuation mean free path and $p_{12}$ is the probability that an interacting particle of group 1 which is thrown out of group 1 by the interaction falls into group 2 .

The observed depth dependence of each appropriate flux ratio (Fig. 6) was fit according to the above equation. The resulting slopes were used to extrapolate flux ratios observed at float altitude to the top of the atmosphere. (Results are given in the next section.)

An estimate of the atmospheric fragmentation spectrum of the iron group $(25 \leq Z \leq 30)$ was obtained by investigating the depth dependence of the ratio $N(25 \leq Z \leq 30) / N\left(Z_{0} \leq Z \leq 24\right)$ for $18 \leq z_{0} \leq 24$, using Eq. (2). Although the accuracy of this determination was limited by statistical uncertainties, the data was consistent with a flat fragmentation spectrum, with $p_{25-30,20-24}=0.25 \pm 0.12$ for the fragmentation of $25 \leq Z \leq 30$ nuclei into $20 \leq Z \leq 24$ nuclei. 


\section{CHARGE COMPOSITION}

For each flight, a least squares fit to the data was used to determine the charge spectrum at the detector. This spectrum was then extrapolated to the top of the atmosphere. In Table II we summarize our results on the charge composition of nuclei with $16 \leq Z \leq 30$, where the results at the top of the atmosphere represent an average over the two flights at $P_{C} \simeq 4.5 \mathrm{GV}$. For comparison, we show also the charge composition at detector level $\left(4.1 \mathrm{~g} / \mathrm{cm}^{2}\right)$ from flight 1970-2. The quoted uncertainties include statistical and charge identification uncertainties and uncertainties in the extrapolation to the top of the atmosphere.

Except for the ratios involving sulfur $(Z=16)$, these results were derived using only events with $\theta \leq 30^{\circ}$ and the data were extrapolated to the top of the atmosphere as described in the previous section. Because of the level of the iron chamber discriminators, we observed nuclei with $Z<17$ only at wide angles. The MH/VH ratio $[(16-19) /(20-30)]$ was derived using data from flight 1970-2 in the angular range $40 \leq \theta<60^{\circ}$, and extrapolated to the top of the atmosphere $\left(.6 \mathrm{~g} / \mathrm{cm}^{2}\right)$ using $\Lambda_{M H}=22.5 \mathrm{~g} / \mathrm{cm}^{2}$ and $p_{V H, M H}=0.15$ (Freier and Waddington, 1968). The sulfur abundance was extrapolated to the top of the atmosphere using the fragmentation parameters of Long (1968). 
TABLE II

RELATIVE ABUNDANCES

\begin{tabular}{lcc}
\hline Charge Ratio & $\begin{array}{c}\text { Top of Atmosphere } \\
\text { Average of Two Fights }\end{array}$ & $\begin{array}{c}4.1 \mathrm{~g} / \mathrm{cm}^{2} \\
\text { Flight } 1970-2\end{array}$ \\
\hline $24 / 26$ & $0.15 \pm 0.03$ & $0.16 \pm 0.02$ \\
$22 / 26$ & $0.14 \pm 0.03$ & $0.16 \pm 0.02$ \\
$20 / 26$ & $0.21 \pm 0.04$ & $0.22 \pm 0.03$ \\
$18 / 26$ & $0.13 \pm 0.03$ & $0.14 \pm 0.02$ \\
$16 / 26$ & $0.27 \pm 0.06^{\dagger}$ & $0.33 \pm 0.06^{*}$ \\
$(27-24) /(25-30)$ & $0.32 \pm 0.04$ & $0.35 \pm 0.02$ \\
$(20-24) /(25-30)$ & $0.45 \pm 0.05$ & $0.50 \pm 0.03$ \\
$(18-20) /(21-30)$ & $0.24 \pm 0.03$ & $0.26 \pm 0.02$ \\
$(16-19) /(20-30)$ & $0.31 \pm 0.05^{\dagger}$ & $0.36 \pm 0.05^{*}$ \\
\hline
\end{tabular}

FFlight 1970-2 only

*At $\sim 6 \mathrm{~g} / \mathrm{cm}^{2}$ 
Figure 7 compares our results for even-z nuclei with those of other recent experiments of good charge resolution. Our results agree well with those of Webber et al. (1971) and Casse et al. (1971). 


\section{ABSOLUTE FLUX}

The absolute flux of relativistic $V H$ nuclei has been determined by a number of workers at various geomagnetic cutoffs and levels of solar activity. The results with the greatest statistical weight until this experiment are those of Webber and Ormes (1967), Freier and Waddington (1968), and von Rosenvinge et al. (1968, see von Rosenvinge, 1970, for a more complete summary of this work). In general, our fluxes are consistently higher than those obtained by these other workers.

Figure 8 and Table III summarize our results. In Table II we have selected data from our flights obtained at the same vertical geomagnetic cutoff as the flights of these other workers. All measurements have been extrapolated to the top of the atmosphere using the value $\Lambda_{V H}=19.7 \pm 1.8 \mathrm{~g} / \mathrm{cm}^{2}$ determined in this experiment. Normalization to a Mt. Washington neutron monitor rate of 2350 was accomplished using the helium regression curve of Webber (1967). The uncertainties are those given by the authors. In the case of von Rosenvinge (1970), they include only statistical uncertainties. Errors quoted on our results include effects of uncertainties in charge identification, in corrections for the few rejected events, and in extrapolation to the top of the atmosphere, as well as the relatively small statistical errors. We note 


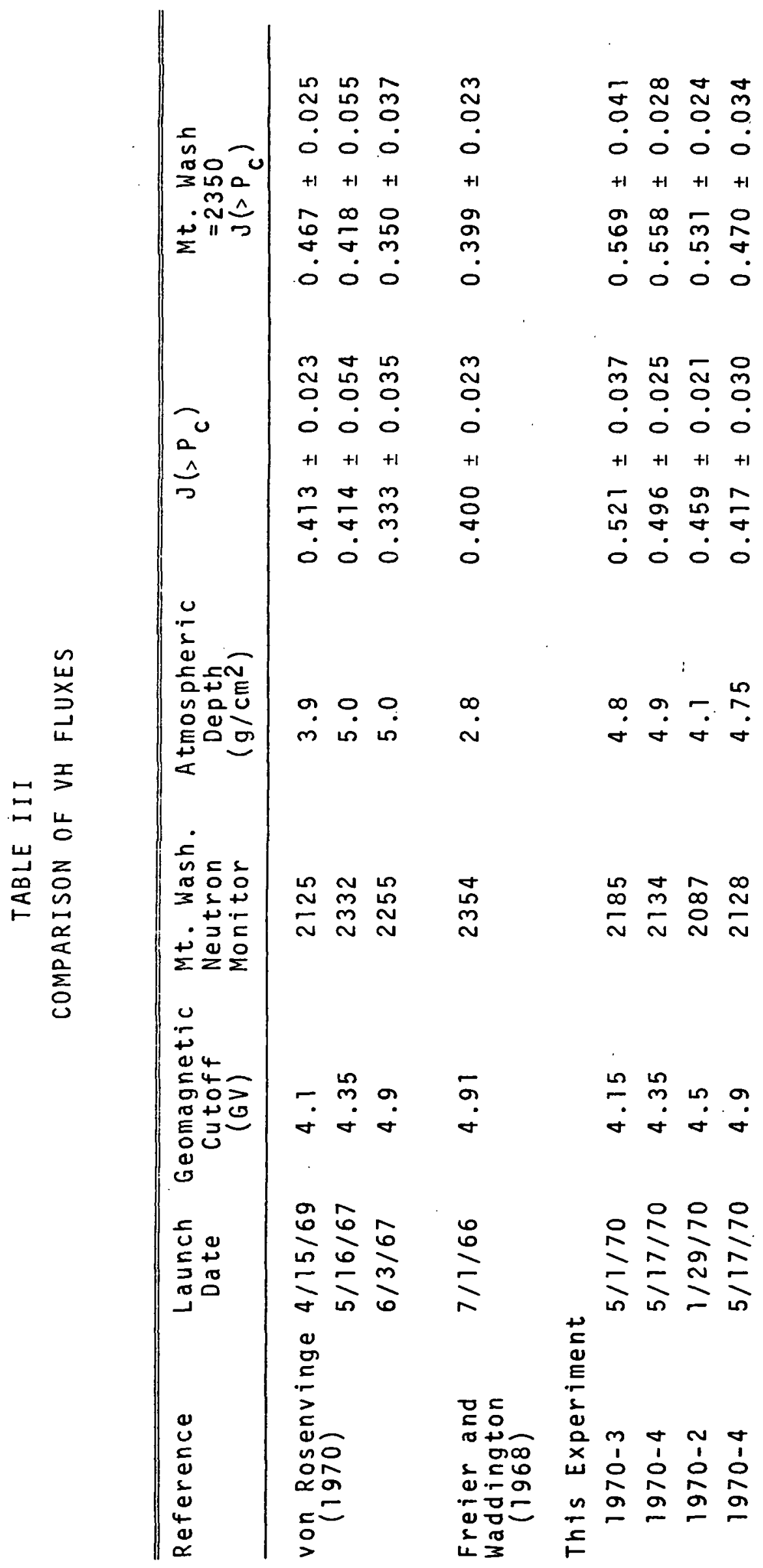


the general self-consistency of our measurements over three flights. We also obtain consistent VH flux measurements over the area of our counter, and at various zenith angles, taking into account the appropriate atmospheric attenuation. This degree of consistency allows upper limits of only a few percent to be placed on the number of out of geometry events that may have been accidentally included in the analysis. We have applied a number of small corrections for the effects of interactions and losses within our counter, but the product of these amounts to less than $4 \%$, within our quoted uncertainty.

We note from Figure 8 that our VH fluxes are systematically about $20 \%$ higher than those of von Rosenvinge. Our disagreement with Freier and Waddington is smaller. Although the fluxes of these other experiments are derived from only a few hundred particles, the disagreement is appparently not of statistical origin.

As the VH charge composition derived from the Freier and Waddington 4.9 GV flight (see Waddington et al., 1970) is significantly different from that found by other workers, it is conceivable that charge identification problems have caused them to find a low VH flux.

Two of the flights of von Rosenvinge involved systematic corrections of the order of $100 \%$ for $V H$ nuclei, the result of losses of events within the detector system (von Rosenvinge, 1970). As no estimate of the uncertainty in this 
correction is included, it is difficult to assess the seriousness of the disparity. A large error in the determination of the residual atmosphere is apparentiy ruled out by the fact that the disagreement between von Rosenvinge and ourselves spans three flights with each detector. As the flight of von Rosenvinge at 4.1 GV occurred at a neutron monitor rate (2125) equal to that of our flight 1970-4 (2128), we can apparently eliminate the possibility of different levels of solar modulation.

It is of interest to look at these discrepancies in terms of the charge composition of the VH group. Von Rosenvinge obtains a $(20-24) /(25-30)$ ratio of 0.61 at the top of the atmosphere from two flights at 4.35 and $4.9 \mathrm{GV}$. We obtain at 4.5 GV a $(20-24) /(25-30)$ ratio of $0.45 \pm 0.05$, in agreement with the recent measurements of Webber et al. (1971) and Casse et al. (1971). We find that our measurements of the (20-24) group flux are in general agreement with von Rosenvinge, thus the discrepancy between our VH flux measurement and that of von Rosenvinge appears to be concentrated in the iron group. Our value for the $M H(16 \leq Z \leq 19)$ flux $\left(0.14 \pm 0.03 \mathrm{~m}^{-2} \mathrm{sr}^{-1} \mathrm{sec}^{-1}\right)$. is also in apparent agreement with von Rosenvinge's value. 


\section{COMPOSITION AT SOURCE}

In this section we present cosmic ray source compositions derived from our measurements. We have calculated the composition which would be observed at earth assuming various source compositions and propagation parameters. The experimentally observed spectrum was then used to place restrictions on the source composition and mode of propagation.

In the "leaky box" model (Goeckler and Jokipii, 1969), it is assumed that the cosmic ray sources are uniformly distributed in space and time, such that an equilibrium is established between creation and loss. The particles are assumed to be confined to the galaxy, although there is a finite probability for escape which can be characterized by a mean life against escape, $T$, commonly referred to as the "leakage lifetime". This steady state model is mathematically equivalent to an exponential path length distribution (Davis, 1960).

In the steady state the number density, $N_{j}$, of particles of type $j$ with a uniform continuous source $Q_{j}$, is then described by

$$
-\frac{N_{j}(x)}{\Lambda_{j}}-\frac{N_{j}(x)}{x}+\sum_{i>j} \frac{N_{i}(x)}{\Lambda_{i}} P_{i j}+Q_{j}=0
$$

where the leakage length, $X$, is $\rho c T$, with $\rho$ the interstellar gas density. 
The fragmentation parameters $\left(p_{i j}\right)$ were calculated from the semiempirical cross-section formula of Shapiro and silberberg (1970) for a representative energy of $2.5 \mathrm{GeV} / \mathrm{n}$, and the $\Lambda$ for a nucleus of atomic number $A$ from

$$
\Lambda=M_{p} / \sigma
$$

where $\quad M_{p}=$ proton mass

and

$$
\sigma \quad=\pi\left(r_{0} A^{1 / 3}\right)^{2}
$$

with

$$
r_{0}=1.2 \times 10^{-13} \mathrm{~cm}
$$

We have assumed a pure hydrogen medium.

Our neglect of energy loss processes is justified provided $(d E / d x) / E \ll \frac{1}{\Lambda}+\frac{1}{X}(G l o e c k l e r$ and Jokipii, 1969). This inequality is satisfied for the relativistic particles we observe. (For $F e^{56}$ nuclei at $P_{c}=4.5 \mathrm{GV},(\mathrm{dE} / \mathrm{dx}) / E \simeq 0.04$ and $\left.\frac{l}{\Lambda}+\frac{1}{X} \simeq 0.6\right)$. The error due to neglect of energy-loss is further minimized since we treat only charge ratios of integral fluxes, over a limited charge region.

In Figure 9 we compare the detailed predictions of the steady state calculation with our observations of even-Z nuclei. We have adjusted the leakage length so that the observed events with $21 \leq Z \leq 24$ are accounted for by interstellar fragmentation of iron. (It is reasonable to assume a very low source abundance for these elements in view of their very low abundance in the solar system, 0.02 of iron. 
(Cameron, 1968)) Assuming a pure iron source (solid line) large discrepancies appear at $Z=16$ and $Z=20$, implying a substantial source abundance of these nuclei. This conclusion is not surprising in view of the pronounced peaks in the experimental charge spectrum (Fig. 4) at calcium and sulfur.

To explain the observed abundances, we require a calcium to iron ratio at the source of $0.12 \pm 0.04$. For sulfur we find $(\mathrm{S} / \mathrm{Fe})_{\text {source }}=0.14 \pm 0.05$. The dotted line in Figure 10 shows the effect of including this amount of calcium and sulfur at the source. Notice that fragmentation of the additional calcium also improves the agreement at argon $(z=18)$.

The leakage length $X \simeq 4 \mathrm{~g} / \mathrm{cm}^{2}$ implied by this comparison is consistent with values that are found to fit the $L / M$ ratio and VVH nuclei. (Gloeckler and Jokipii, 1969; Cowsik et al.,1970; Shapiro et al., 1970; Fowler et al., 1970).

We have also performed propagation calculations for the simple "slab model". The observed $(21-24) /(25-30)$ ratio sets an upper limit of $x_{0}=1.85 \pm 0.2 \mathrm{~g} / \mathrm{cm}^{2}$ to the amount of interstellar hydrogen traversed. Although this small value of $x_{0}$ rules out the slab as a generally applicable model (because the observed ratio of light to medium nuclei requires $x_{0}$ of 3 to $5 \mathrm{~g} / \mathrm{cm}^{2}$ ) it is still instructive to derive source abundances over a limitedinterval of charges using this model. 
Table IV summarizes the inferred source composition and compares the result with the Cameron (1968) solar system abundances. These cosmic ray results depend only weakly on the $(21-24) /(25-30)$ ratio at the source. For example, if $(21-24) /(25-30)_{\text {source }}=0.1$ we find $\mathrm{Ca} / \mathrm{Fe}=0.15 \pm 0.04$ and $S / F e=0.18 \pm 0.05$ on the steady state model. We conclude that for either the $\operatorname{slab}\left(x_{0} \simeq 2 \mathrm{~g} / \mathrm{cm}^{2}\right)$ or the steady state model $\left(X \simeq 4 \mathrm{~g} / \mathrm{cm}^{2}\right)$ the observed high energy charge spectrum for $16 \leq Z \leq 26$ is consistent with a source composed primarily of iron, with smaller amounts of calcium and sulfur. In fact the relative abundances of sulfur, calcium, and iron at the source are essentially the same in either model. The remaining abundances are consistent with a fragmentation origin and these elements are not required in any large concentration at the source, although abundances of a few percent that of iron are not excluded.

Several other authors have recently considered the source abundances of cosmic ray nuclei in this charge region, including Shapiro and Silberberg (1970), von Rosenvinge (1970), Webber et al. (1971), Cartwright et al. (1971), and Shapiro et al. (1971). There now appears to be general agreement among all workers on the abundance of sulfur at the source. Shapiro et al. (1971), using a weighted average of data from many experiments published prior to 1971, have recently revised their calculations (Shapiro and Silberberg, (1970), and now include small and roughly equal amounts of argon and 
TABLE IV

ABUNDANCES RELATIVE TO IRON

Cosmic Ray Source

Calcium

sulfur

$$
\begin{aligned}
& (\mathrm{s} l a b \text { model, } \\
& x_{0}=1.85 \mathrm{~g} / \mathrm{cm}^{2} \text { ) } \\
& \text { Cosmic Ray Source } \\
& (1 \text { eaky box model, } \\
& \left.x \approx 4 \mathrm{~g} / \mathrm{cm}^{2}\right)
\end{aligned}
$$

$0.13 \pm 0.04$

$0.16 \pm 0.05$

Solar System

(Cameron, 1968)

0.08

0.57 
calcium at the source, $(\mathrm{A} / \mathrm{Fe}=\mathrm{Ca} / \mathrm{Fe}=0.09 \pm 0.09)$. The results of our investigation indicate a slightly higher source abundance of calcium, and we definitely find $\mathrm{A}<\mathrm{Ca}$, in general agreement with von Rosenvinge (1970), and Webber et al. (1971). Earlier experimental measurements showed a sizeable Cr/Fe ratio at both high and low energies (Mathiesen et al., 1968, Waddington et al., 1970; Lezniak et al., 1970; Dayton et al., 1970; Price et al., 1970; Garcia-Munoz and Simpson, 1970), forcing the conclusion that substantial chromium existed at the source (Shapiro and Silberberg, 1970, von Rosenvinge, 1970, Longerfelter and Ramaty, 1971). More recent measurements with better resolution and statistics show a much lower flux of chromium (Webber et al., 1971; Casse et al., 1971). The chromium abundance observed in this experiment is low enough to conclude that it is principally of secondarry origin, in agreement with the conclusions of Shapiroet al. (1971), and Webber et al. (1971). At low energies, the recent satellite measurements of Cartwright et al. (1971) at 20-300 MeV/n imply a source composition with $\mathrm{Ca} / \mathrm{Fe}=0.1$ and $\mathrm{S} / \mathrm{Fe}=0.16$ in agreement with that found here.

We conclude that the trend of the most recent experimental and theoretical work favors finite source abundances of calcium and sulfur, with all other elements (with $Z>16$ ) less than $10 \%$ of $\mathrm{Fe}$ at the source. Future work will concentrate on some of these less abundant elements. 
Comparison between our cosmic-ray source results and the Cameron solar system abundances indicates a slight cosmic-ray excess in calcium and a marked cosmic-ray deficiency in argon and sulfur. We note, however, that the solar system argon abundance is very uncertain (as with other volatile elements) and Cameron's abundance for $A^{36}$ is simply an interpolation between observed abundances of $\mathrm{S}^{32}$ and $\mathrm{Ca}^{40}$. The low cosmicray sulfur abundance appears to represent a real disparity with the solar system.

We consider it unlikely that the differences we find between the cosmic-ray source and solar-system composition are a result of propagation effects. More likely, the explanation is to be found in the environmental conditions under which these nuclei were synthesized. It now appears that solar-system elements in this charge region were synthesized under explosive conditions in massive stars, as evidenced by the dramatic success of explosive nucleosynthesis theory in matching the solar-system abundances (Arnett and Clayton, 1970). With the cosmic ray elemental (although not yet isotopic) abundances now known to an accuracy comparable to the solar-system abundances, it should soon be possible to identify the environment where these nuclei originated. 


\section{ACKNOWLEDGEMENTS}

The authors gratefully acknowledge the technical efforts of J.W. Epstein, A. J. Taylor and K. E. Krippner for essential technical support in design, fabrication and operation of the instrument. We are also indebted to the crew of the NCAR balloon flight station in Palestine, Texas for their splendid support of the balloon flights. This work was supported in part by NASA grant NGR-26-008-001 and in part by McDonnell Douglas Corporation, Independent Research and Development Program. One of us (RAM) was partially supported by an NSF graduate fellowship. One (JIF) was partially supported by a Rockefellar Foundation fellowship. 


\section{REFERENCES}

Arnett, W. D. and Clayton, D. D.: 1970, Nature 227, 780 .

Binns, W. R., Fernandez, J. I., Israel, M. H., Klarmann, J., and Mewaldt, R. A.: 1971, 12 th International Conference on Cosmic Rays, Hobart, 1971, Conference Papers 1, 260.

Bradt, H. L. and Peters, B.: 1950, Phys. Rev. $80,943$.

Cameron, A. G. W.: 1968, in L. H. Ahrens (ed.), Origin and Distribution of the Elements, Pergamon Press, Oxford.

Cartwright, B. G., Garcia-Munoz, M., and Simpson, J. A.: 1971 , 12th International Conference on Cosmic Rays, Hobart, 1971 , Conference Papers 1, 215.

Casse, M., Koch, L., Lund, N., Meyer, J.P., Peters, B., Soutoul, A., and Tandon, S. N.: 1971, 12 th International Conference on Cosmic Rays, Hobart, 1971, Conference Papers 1, 241 .

Cleghorn, T. F., Freier, P. S., and Waddington, C. J.: 1968, Can. ‥ Phys. $\underline{46}$, S 572 .

Cowsik, R., Pal, Yash., Tandon S. N., and Verma, R. P.: 1970, Acta Physica Hung. 29, Supp 1 1, 439

Daniel, R. R. and Durgaprasad N.: 1962, Nuovo Cimento Suppl. $\underline{23}, 82$.

Davis, L., Jr.: 1960 , Proceedings of the Moscow Cosmic Ray Conference 3,220 .

Dayton, B., Lund, $\mathrm{N}_{.}$, and Risbo, T.: 1970, Acta Physica Hung: 29, Suppl. 1, 385 .

Epstein, J. W., Fernandez, J. I., Israel, M. H., Klarmann, J., and Mewaldt, R. A.: 1971, Nuc. Inst. and Methods 95, 77 .

Fernandez, J. I.: 1972, Ph.D. Thes is, Washington University.

Fowler, P. H., Clapham, V. M., Cowen, V. G., Kidd, J. M., and Moses, R. T.: 1970, Proc. Roy. Soc. Lond. A318, 1.

Freier, P. S. and Waddington, C. J.: 1968, Phys. Rev. 175, 1641 .

Garcia-Munoz, M. and Simpson, J. A.: 1970, Acta Physica Hung. 29, Supp 1. 1, 317 . 
Gloeckler, G. and Jokipii, J.R.: 1969, Phys. Rev. Letters $\underline{22}, 1448$.

Lezniak, J. A., von Rosenvinge, T. T., and Webber, W. R.: 1970, Acta Physica Hung. 29, Supp 1. 1, 375.

Lingenfelter, R. E. and Ramaty R.: 1971, 12th International Conference on Cosmic Rays, Hobart, 1971, Conference Papers 1,377 .

Long, C. E.: 1968, M. S. Thesis, University of Minnesota.

Mathieson, 0., Long, C. E., Freier, P. S., and Waddington, C. J.: 1968, Can. ‥ Phys. 46, S 583 .

Mewaldt, R. A.: 1971, Ph.D. Thesis, Washington University.

Ormes, J.F. and Balasubrahmanyan, V. K.: 1970, Acta Physica Hung. 29, Suppl. 4, 397.

Price, P. B., Peterson, D. D., Fleischer, R. L., O'Ceallaigh, C., O'Sullivan, D., and Thompson, A.: 1970, Acta Physica Hung. 29, Supp1. 1,417.

Von Rosenvinge, T. T.: 1970, Ph.D. Thesis, University of Minnesota.

Von Rosenvinge, T. T., Webber, W. R., and Ormes, J. F.: 1969, Astrophys. Space Sci. $\underline{5}, 342$.

Shea, M. A., Smart, D. F., and McCall, J. R.: 1968, Can. J. Phys. 46, S. 1098 .

Shapiro, M. M. and Silberberg, R.: 1970, Ann. Rev. Nucl. Sci. 20, 323 .

Shapiro, M. M., Silberberg, R. and Tsao, C. H.: 1970, Acta Physica Hung. 29, Supp 1. 1, 471 .

Shapiro, M. M., Silberberg, R., and Tsao, C. H.: 1971, 12th International Conference on Cosmic Rays, Hobart, 1971 , Conference Papers 1, 221.

Waddington, C. J.: 1960, Phil. Mag. $5,311$.

Waddington, C. J., Freier, P. S., and Long, C. E.: 1970, Acta Physica Hung. 29, Supp 1. 1, 367. 
Webber, W. R.: 1967, in Handbuch der Physik 46-2, SpringerVerlag, Berlin, p. $1 \overline{81}$.

Webber, W. R. and Ormes, J. F.: 1967, J. Geophys. Res. 72, 5957 .

Webber, W. R., Damle, S. V., and Kish, J.M.: 1971, 12th International Conference on Cosmic Rays, Hobart, 1971, Conference Papers 1,229. 


\section{FIGURE CAPTIONS}

1. Schematic cross-section of the detector system.

2. Square root of ionization vs square root of Cerenkov pulse-heights for various nuclei.

3. Cross-plot of square root of ionization vs square-root of Cerenkov pulse-height for 2000 events.

4. Charge histogram. At charge 16 and below the number of events is depressed due to electronic threshold. Arrows above charge 30 indicate single events.

5. Atmospheric attenuation of $V H$ nuclei and of iron nuclei. Symbols indicate flights. $\Delta-1970-2, X-1970-3,0-1970-4$.

6. Atmospheric depth dependence of various charge ratios. The data points are averaged over the three flights.

7. Comparison of abundance measurements for even-Z nuclei at the top of the atmosphere.

8. Comparison of VH flux measurements. All measurements have been extrapolated to the top of the atmosphere using $\Lambda_{V H}=19.7 \mathrm{~g} / \mathrm{cm}^{2}$ and normalized to a Mt. Washington neutron monitor rate of 2350 .

9. Comparison between observed abundances and abundances calculated in steady state model for a pure iron source and for a source with calcium and sulfur abundances $12 \%$ and $14 \%$ of iron respectively. 


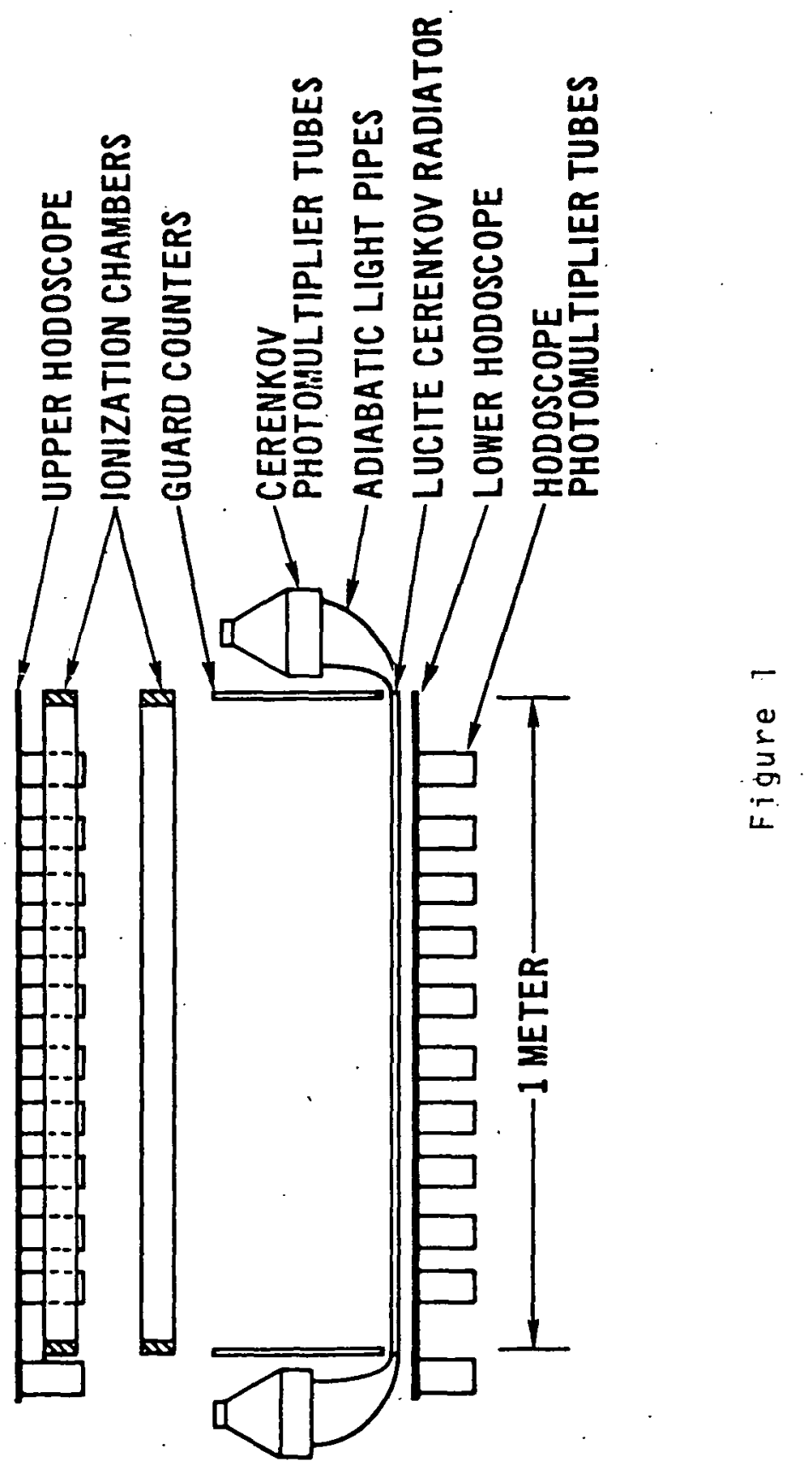




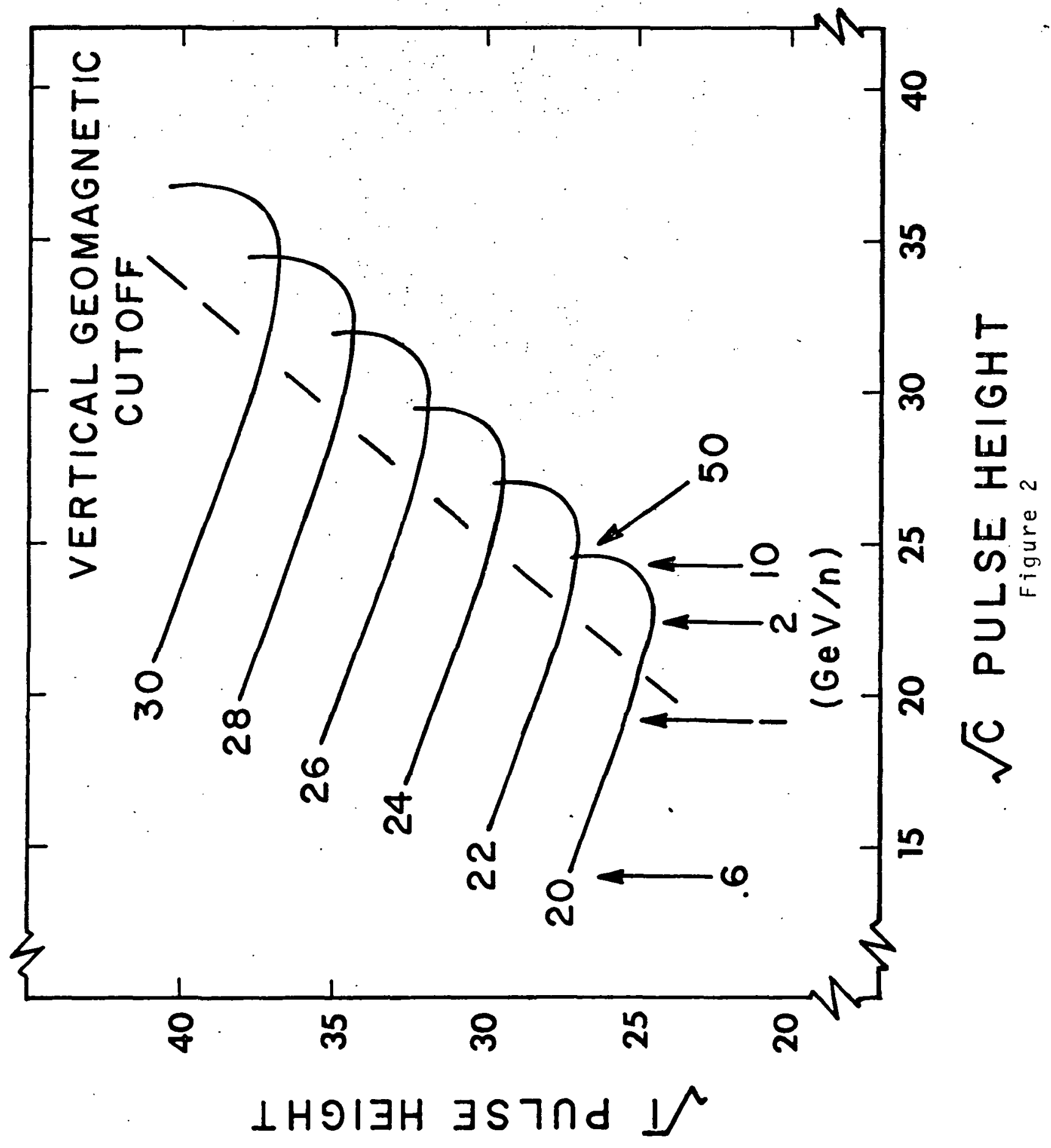




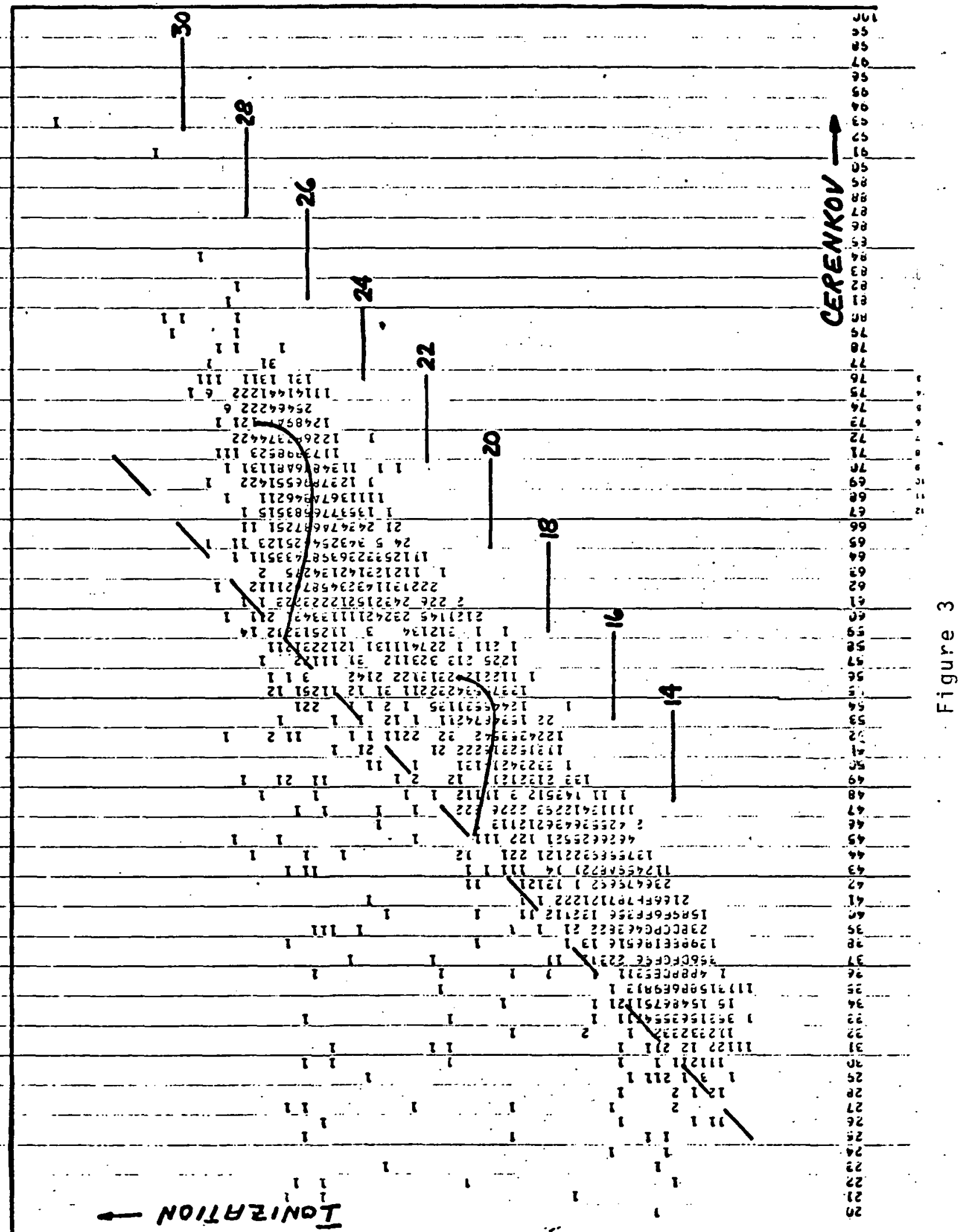

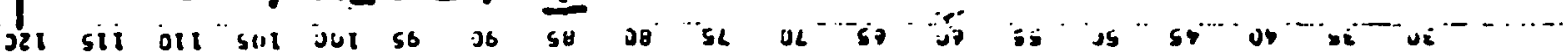




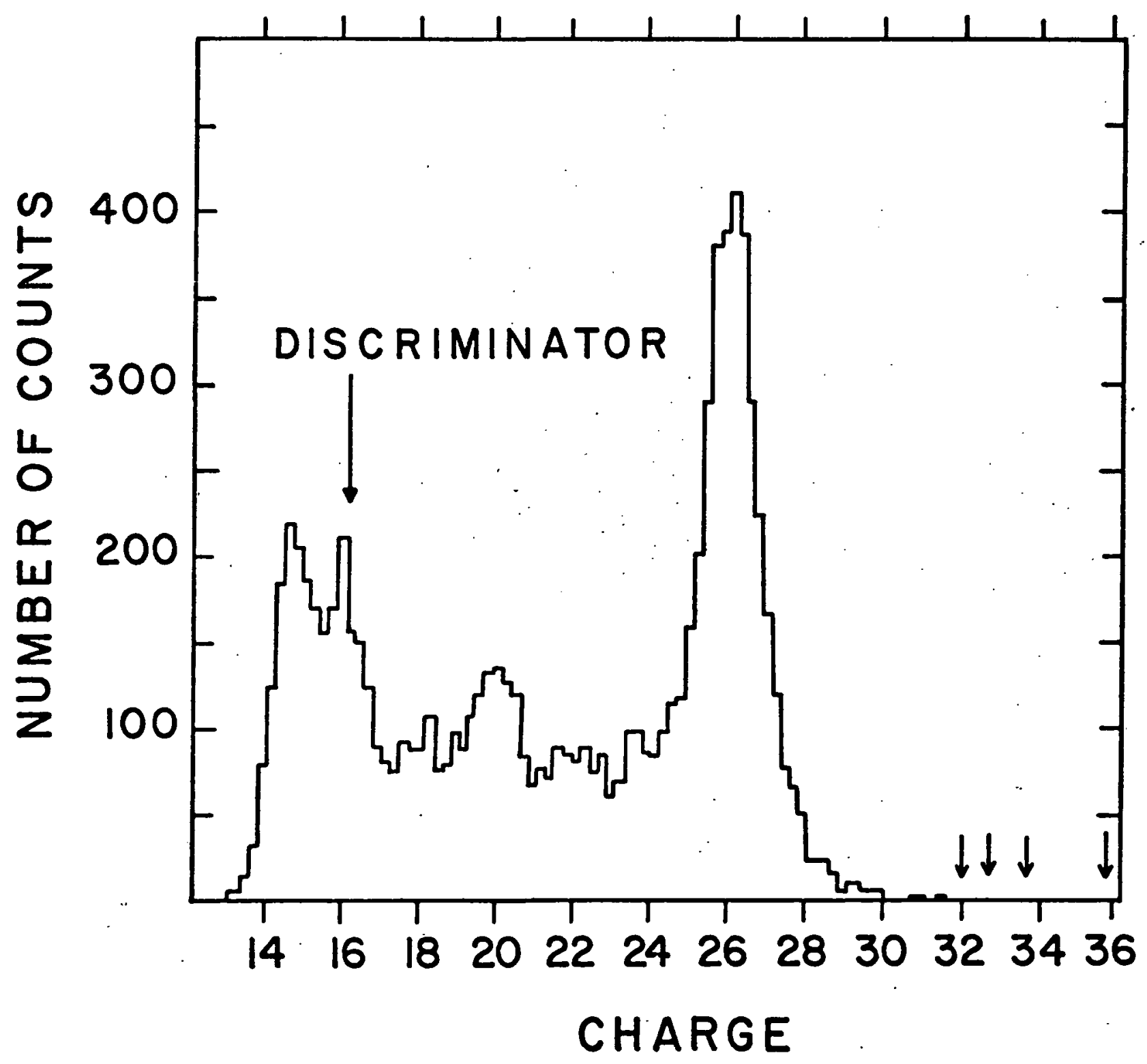

Figure 4 


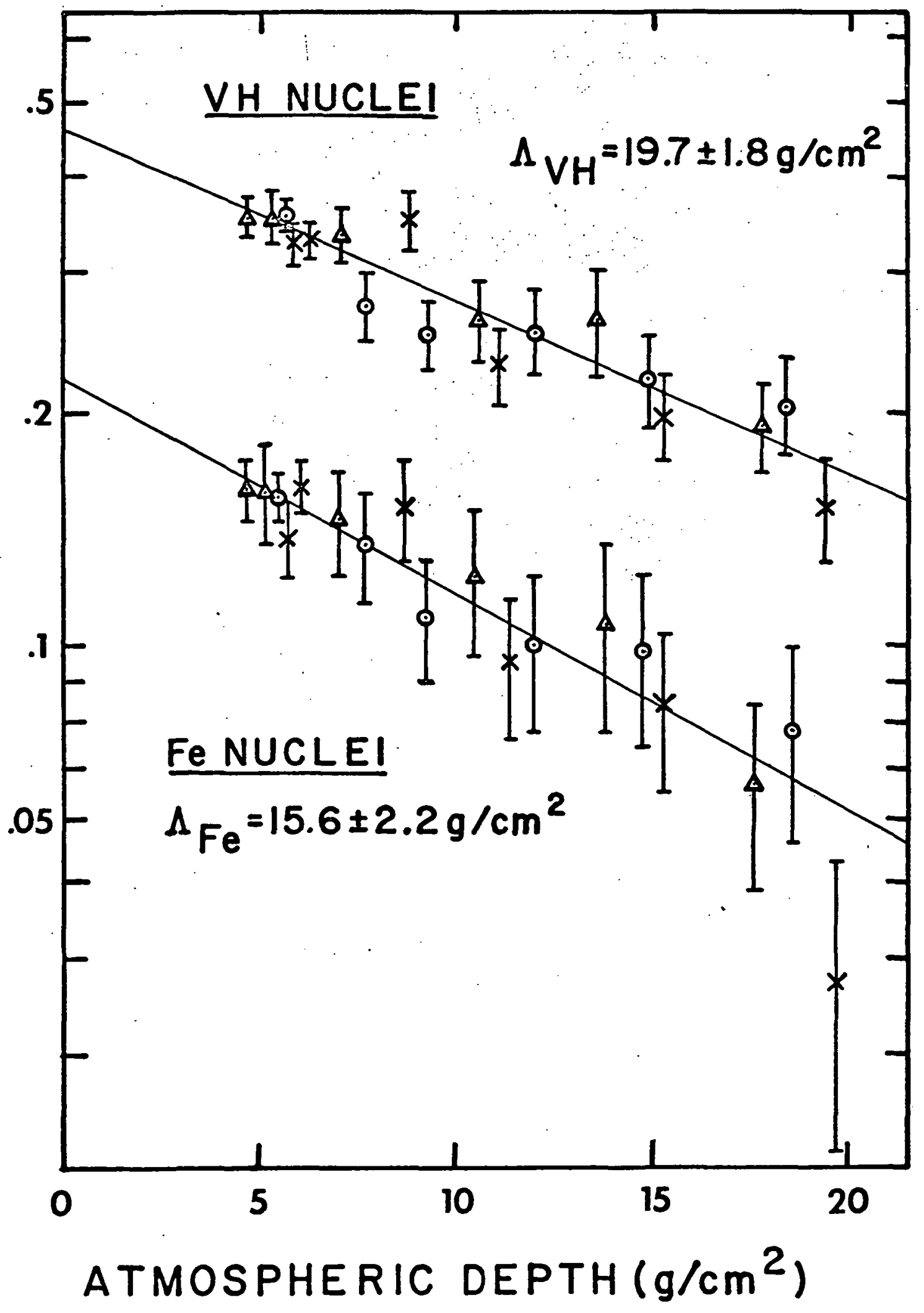

Figure 5 


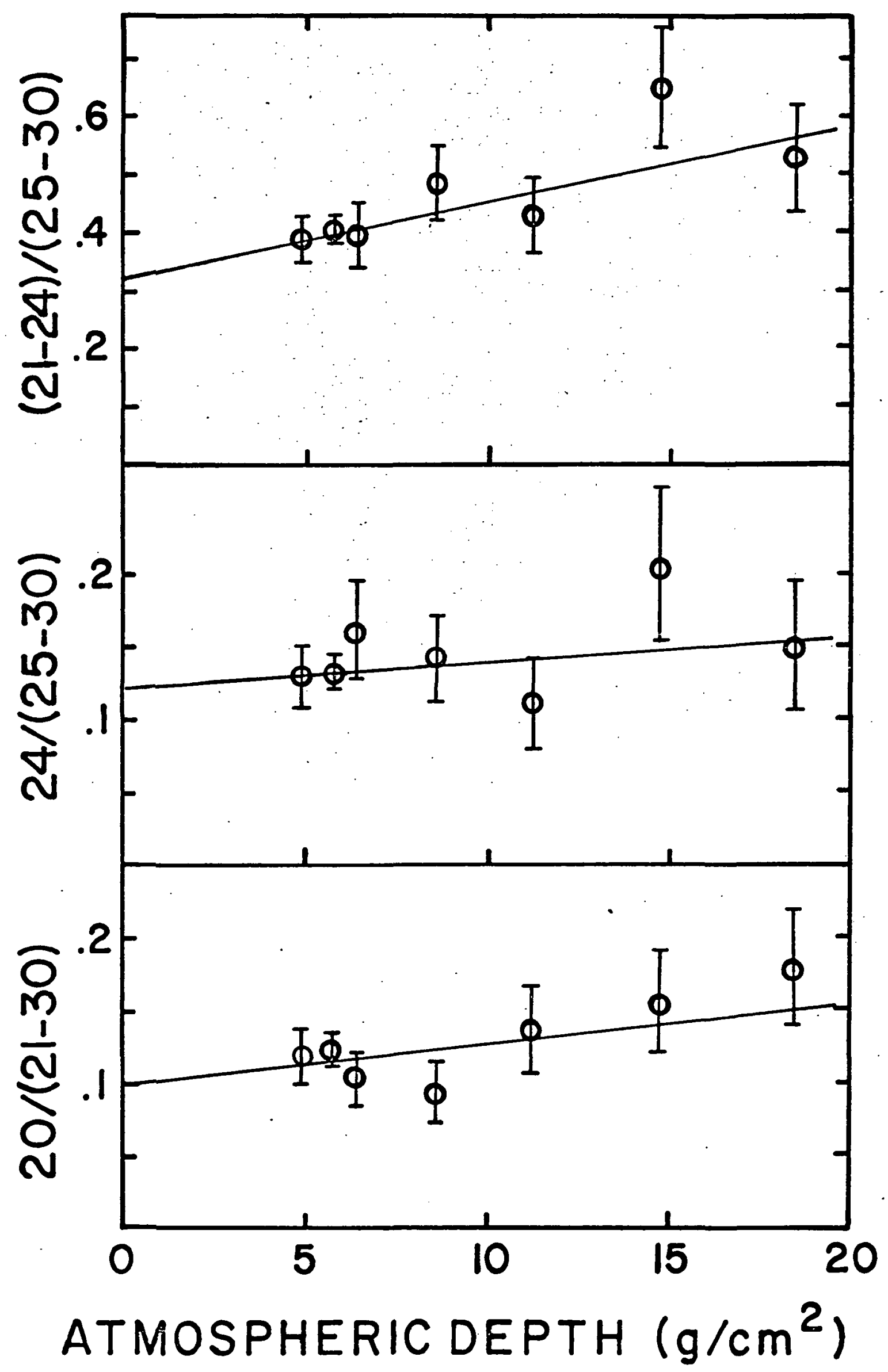

Figure 6 


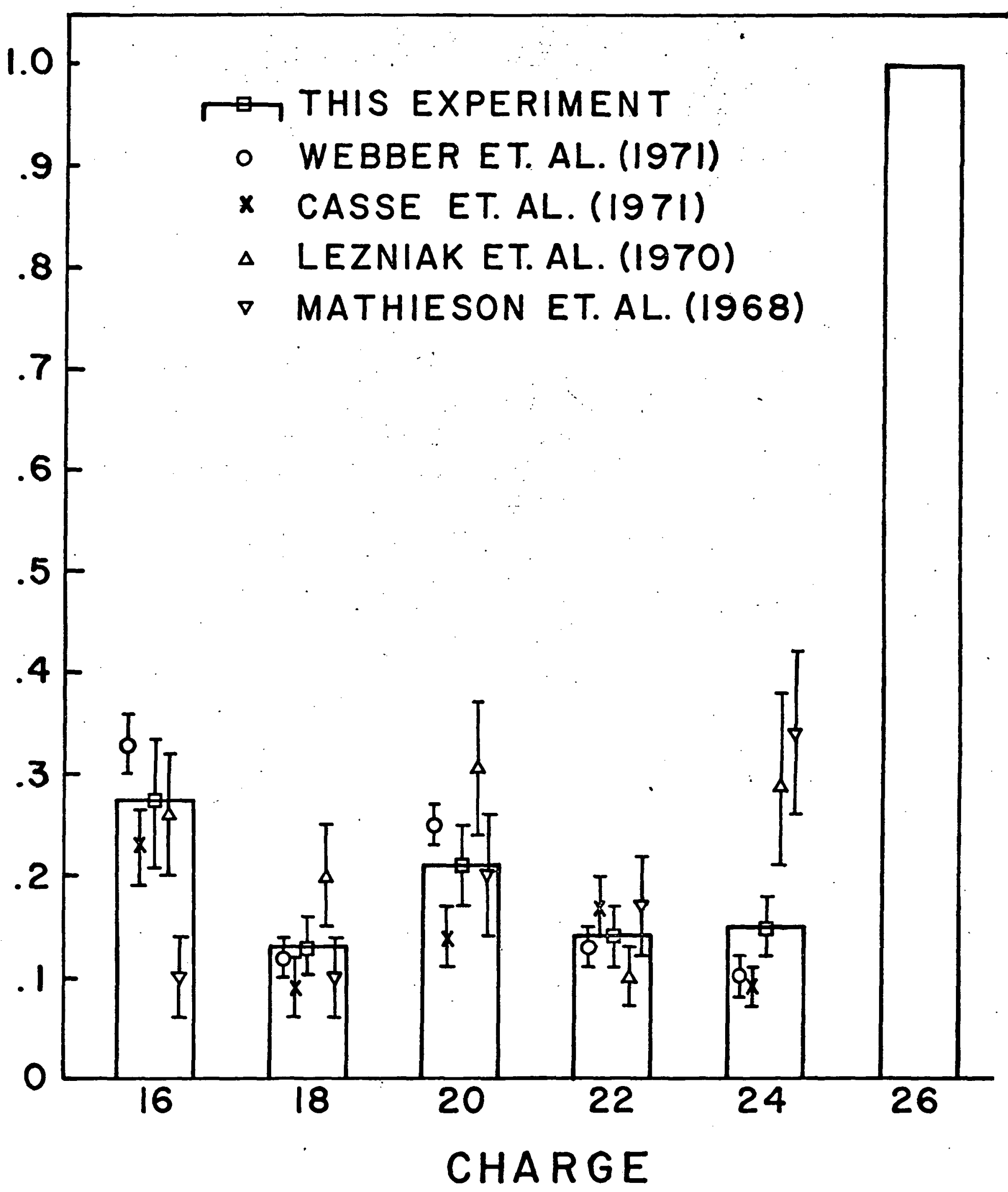

Figure 7 


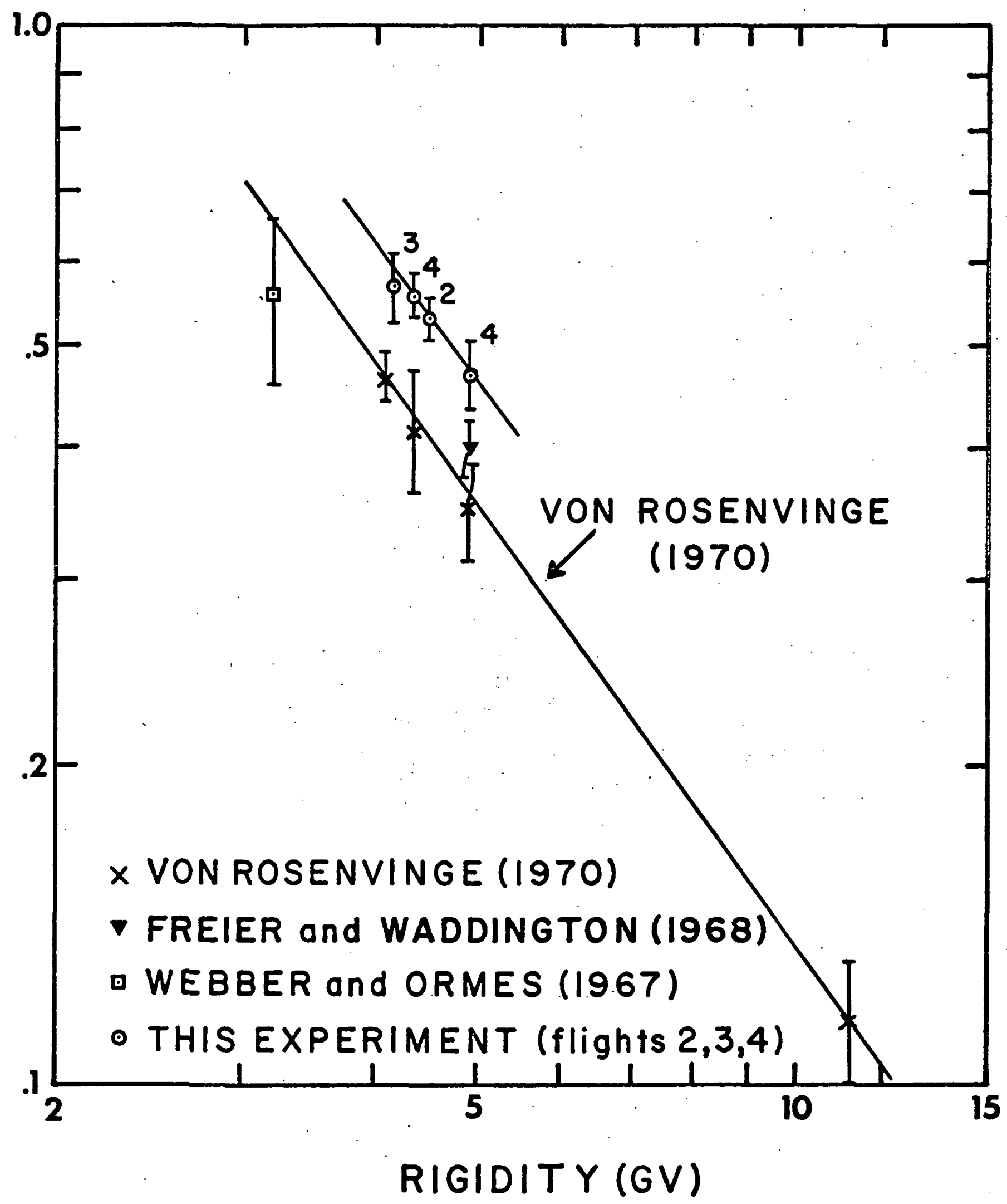

Figure 8 


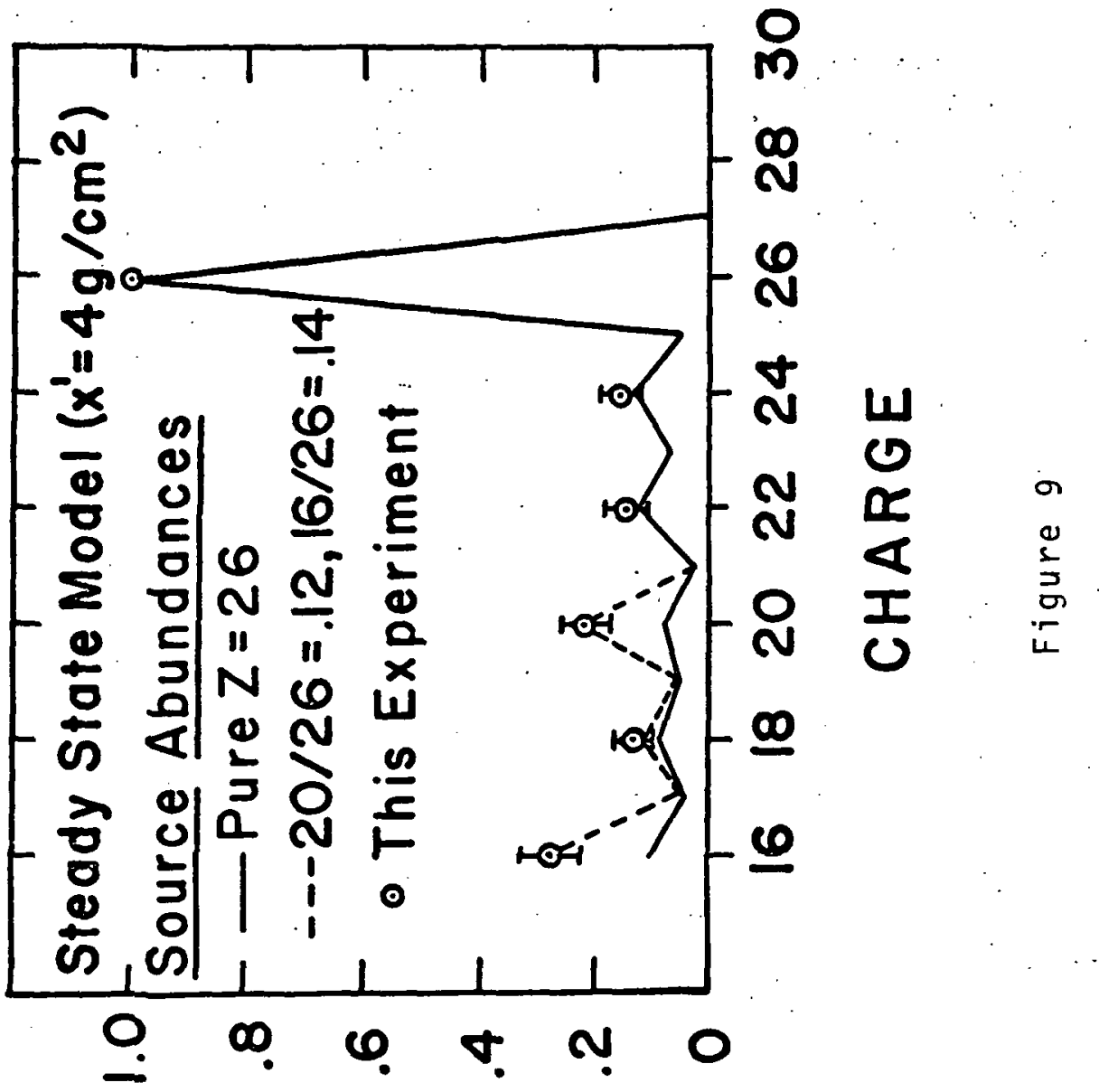

$\exists \supset N \forall O N \cap Q \forall \exists \wedge I \perp \forall \neg \exists y$ 